\title{
Ground-based Support of Comet Nuclei Space Missions
}

\author{
Jana Pittichová \& Karen J. Meech \\ Institute for Astronomy, University of Hawaii, 2680 Woodlawn Dr., \\ Honolulu, HI 96822, USA
}

\begin{abstract}
There are currently five space missions en route to or planned for comet encounters. It is important for the success of each mission that its target be characterized as fully as possible prior to the encounter. This progress report analyses initial photometric results for 11 comet nuclei for the Stardust, Rosetta, Deep Impact, CONTOUR, Deep Space 1, and the proposed Comet Nucleus Sample Return missions from a database of comet CCD images. Our 17-year database contains more than 20,500 images of $\sim 160$ comets taken during $>1,500$ observing hours (Pittichová \& Meech 2000). We are undertaking a program to observe these 11 comets over a wide range of heliocentric distances to obtain information about their behavior and composition that will be particularly useful to the space missions.
\end{abstract}

\section{Introduction}

It is known that cometary nuclei contain a large amount of water and organic molecules, which are very important for life. The primeval Earth was hit by a large number of these bodies and enriched by water and other important molecules. Cometary nuclei played a significant role in the formation of life on Earth. Five scheduled and proposed NASA Discovery Programs, for which comet nuclei are primary targets, will document the importance of cometary research for our knowledge about the origin and evolution of the Solar system, individual planets, and life on Earth. Knowledge of the nucleus size, albedo, spin state, and characterization of the dust and gas production rates are all important for mission planning and success. Although comet nucleus samples don't yet exist, we can obtain important information about comet behavior and composition (nucleus size, rotation period, and thermal emission of dust) from the photometric analysis of ground-based data. The specifics of the orbital geometry, number of images, percentage of photometry reduction done and statement of which type of information (rotation period, nucleus size and dust-dynamical model) we can obtain from each comet, are shown in Table 1.

Deep Space 1 - The mission was launched in October 1998 and encountered comet 19P/Borrelly during September 2001. This mission was testing 12 new technologies for future missions, such as ion drive rocket engines, new solar panels, and autonomous navigation systems. We can use the mission optical data to study the nucleus size, albedo, and rotation of the comet. 
Stardust - This mission launched in September 1999 will encounter comet $81 \mathrm{P} /$ Wild 2 in January 2004. This is the fourth Discovery mission and will be the first return of cometary material back to Earth (January 2006). The spacecraft will fly through the cloud of dust that surrounds the comet nucleus and will collect small pieces of this in a special low-density silica aerogel (Brownlee et al. 2000).

CONTOUR - This mission, launched in July 2002, was to have encountered at least two comets, 2P/Encke and 73P/Schwassmann-Wachmann 3 (SW 3 ) in November 2003 and June 2006, during their peaks of activity, while close to Sun. Unfortunately, on August 14, 2002 during the departure from Earth orbit, the spacecraft apparently broke into three pieces and communication was lost.

Rosetta - This mission will be launched in January 2003 to encounter comet 46P/Wirtanen in 2013. The primary goal will be to orbit the comet, obtain extensive data about its nucleus, environment, and surface characteristics, and eventually to land a small probe on the surface. Ground-based data will help us to study nucleus physical characteristics, size, albedo, rotation period, and thermal emission of dust. The secondary mission will be to fly by asteroid 4979 Otawara in July 2006 and asteroid 140 Siwa in July 2008.

Deep Impact - This mission will launch in January 2004 and will encounter comet $9 \mathrm{P} /$ Tempel 1 in July 2005. This is the eighth Discovery mission and the first one to conduct an experiment interacting with the target. The spacecraft will deliver a $370-\mathrm{kg}$ projectile to the comet to excavate a large crater. The excavation down to unaltered material will enable us to make inferences about the chemical and physical conditions in early solar system. We will be able to learn about cratering processes in small bodies and the mechanical properties of cometary surface layers.

CNSR - The Comet Nucleus Sample Return mission is a proposed mission that will fly by a comet and bring a dust sample back to Earth for complete analysis. There are several cometary candidates: comet $46 \mathrm{P} / \mathrm{Wirtanen}, 22 \mathrm{P} / \mathrm{Kopff}$, 16P/Brooks 2, 104P/Kowal 2, 67P/Churyumov-Gerasimenko.

\section{Analysis}

The optical imaging database contains more than 20,500 comet CCD images obtained during 17 years with different telescopes but mostly with the University of Hawaii 2.2-m telescope on Mauna Kea. The steps in the analysis of our data included the following:

- We used a standard photometry routine with different circular aperture radii for the extraction of the flux from CCD images.

- Sky background flux was determined from an annulus centered on the non-active comet, and for active ones, from area around the comet not contaminated by comae.

- We chose an optimum aperture radius of $2^{\prime \prime}-4^{\prime \prime}$ to get a nucleus brightness and minimize the contamination from the surrounding comae. 
- We measured the magnitudes of 20-30 field stars per frame to do differential photometry to determine rotation periods precisely.

- The measured magnitudes of the comet were corrected for extinction using standard stars and for frame-to-frame extinction variations.

Table 1. Completed Photometry for the Comet Datasets.

\begin{tabular}{lccccccc}
\hline \hline $\begin{array}{l}\text { Comet } \\
\text { Name }\end{array}$ & $\begin{array}{c}r \text { Range } \\
(\mathrm{AU})\end{array}$ & $\begin{array}{c}\Delta \text { Range } \\
(\mathrm{AU})\end{array}$ & $\begin{array}{c}\text { \# of } \\
\text { Images }\end{array}$ & $\begin{array}{c}\text { Done } \\
\%\end{array}$ & Rot. & Nuc. & Dust \\
\hline 6P/d'Arrest & $1.7-5.6$ & $1.2-4.8$ & 191 & 90 & No & Yes & Yes \\
19P/Borrelly & $1.5-21$. & $0.5-2.3$ & 38 & 87 & No & Yes & Yes \\
16P/Brooks 2 & $1.9-3.6$ & $1.0-3.1$ & 123 & 97 & No & Yes & Yes \\
2P/Encke & $1.9-4.1$ & $1.9-3.2$ & 248 & 76 & Yes & No & Yes \\
22P/Kopff & $1.6-5.4$ & $0.6-5.7$ & 431 & 31 & No & Yes & Yes \\
104P/Kowal 2 & 5.0 & 4.0 & 2 & 0 & No & No & No \\
73P/SW 3 & 5.1 & 5.2 & 2 & 0 & No & No & No \\
9P/Tempel 1 & $2.2-4.6$ & $2.9-3.7$ & 512 & 82 & Yes & Yes & Yes \\
81P/Wild 2 & $1.6-5.3$ & $0.9-4.7$ & 461 & 95 & Yes & Yes & Yes \\
107P/Wilson- & & & & & & & \\
Harrington & $1.7-4.3$ & $1.3-4.0$ & 27 & 72 & No & Yes & No \\
46P/Wirtanen & $1.6-5.1$ & $1.5-4.3$ & 92 & 98 & No & Yes & Yes \\
\hline
\end{tabular}

\section{Results}

- Optical imaging allows us to estimate the dust production versus heliocentric distance, $r$, and suggest at which distance a comet began active, as you can see in Fig. 2.

- We estimated the upper limit of nucleus radius (Table 2), $R_{n}$ based on the distant comet photometry and assumed the geocentric $\Delta$, and heliocentric distances and geocentric albedo $p_{v}=0.04$,

$$
p_{v} R_{n}^{2}=2.24 \times 10^{22} r^{2} \Delta^{2} 10^{0.4\left(m_{\odot}-m\right)}
$$

where $m_{\odot}$ is the solar magnitude, and $m$, the observed magnitude.

- From comet nucleus brightness changes we were able to determine preliminary rotational light curves for several comets, shown in Fig. 1, and fit a simple sine curve to phase to its rotation period, shown in Fig. 3, and these will also yield information about axis ratios.

- We will use Finston-Probstein dust-dynamical models to obtain informations about the onset and cessation of comet activity, the relative grain velocity distributions, and particle sizes. 

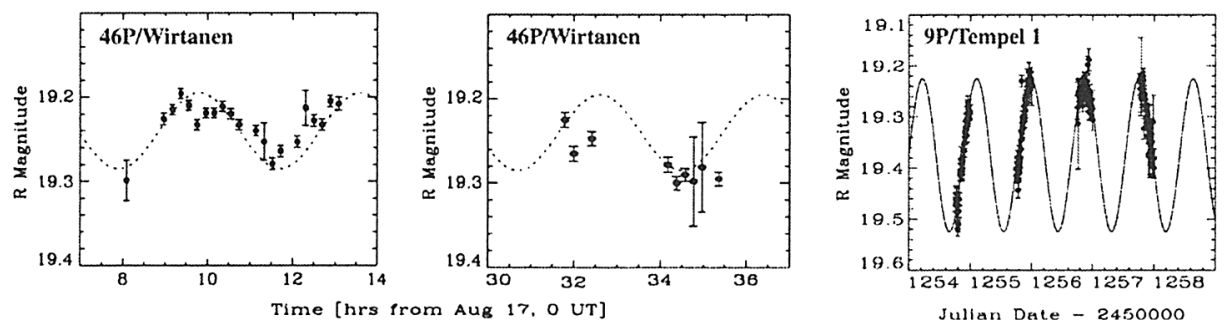

Figure 1. $R$-band rotational light curve of Comet $46 \mathrm{P} / \mathrm{Wirtanen}$ from August 17, $1996 \mathrm{UT}$, through a $2 .{ }^{\prime \prime} 5$ radius aperture (Meech et al. 1997) and Comet 9P/Tempel 1 from March 17-20, 1999 UT, through a 2. .0 (Meech et al. 2000).
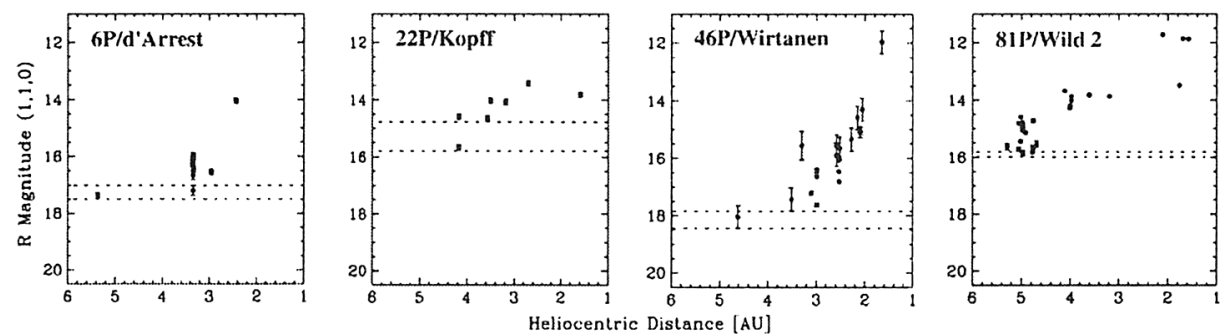

Figure 2. $R$-band data of Comet $6 \mathrm{P} / \mathrm{d}$ 'Arrest and 22P/Kopff from 1988-1997 (Meech et al. 2002), 46P/Wirtanen on 1998-1999 and 81P/Wild 2 from 1999-2000 (Pittichová \& Meech 2001), reduced to unit $r, \Delta$, and zero phase plotted versus $r$. The dotted lines represent the likely brightness range for the bare nucleus.
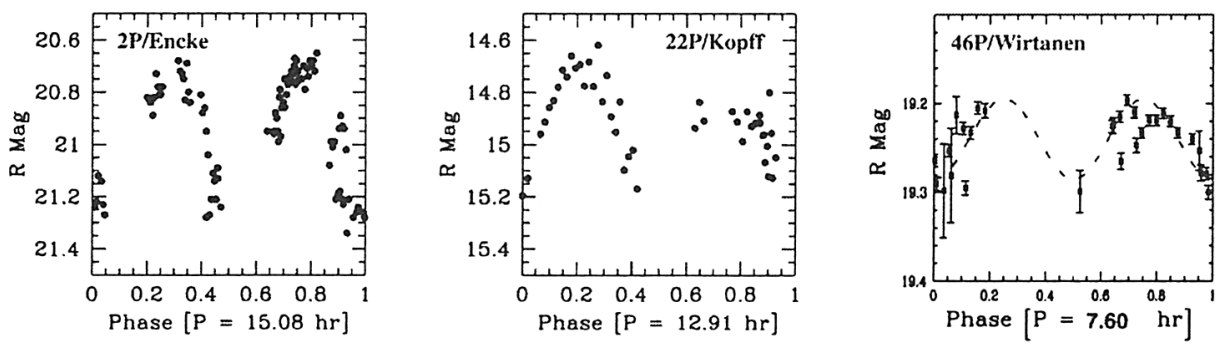

Figure 3. $R$-band data of Comet 2P/Encke from May 13, 1991 (Jewitt \& Meech 1987) phased to the rotation period of 15.08 hours, $22 \mathrm{P} / \mathrm{Kopff}$ from January 4, 1992, phased to the rotation period of 12.91 hours and 46P/Wirtanen from August 17, 1996, phased to the rotation period of 7.60 hours. 
Table 2. Nucleus Size Estimates from Distant Comet Photometry.

\begin{tabular}{lcccc}
\hline Comet Name & $R_{n}(\mathrm{~km})$ & $p_{v}$ & $r(\mathrm{AU})$ & $\Delta(\mathrm{AU})$ \\
\hline 6P/d'Arrest & 1.75 & 0.04 & 5.584 & 4.740 \\
16P/Brooks 2 & 0.99 & 0.04 & 3.571 & 2.600 \\
2P/Encke & 2.40 & 0.04 & 4.072 & 3.240 \\
22P/Kopff & 1.52 & 0.04 & 4.728 & 3.875 \\
9P/Tempel 1 & 2.80 & 0.04 & 4.482 & 3.525 \\
81P/Wild 2 & 2.00 & 0.04 & 5.055 & 4.143 \\
107P/Wilson-Harrington & 2.00 & 0.04 & 4.198 & 3.997 \\
46P/Wirtanen & 0.60 & 0.04 & 3.109 & 3.583 \\
\hline \hline
\end{tabular}

\section{References}

Brownlee, D. E., et al. 2000, M\&PS, 35, A35

Jewitt, D., \& Meech, K. J. 1987, AJ, 93, 1542

Meech, K. J., Bauer, J. M., \& Hainaut, O. R. 1997, A\&A, 326, 1268

Meech, K. J., et al. 2000, in Bioastronomy 99 Conf. Proc., 213, A New Era in the Search for Life, ed. G. A. Lemarchand \& K. J. Meech (San Francisco: ASP), 235

Meech, K. J., et al. 2002, in preparation

Pittichová, J., \& Meech, K. J. 2000, BAAS, 32, no. 3, abstract no. 37.04

Pittichová, J., \& Meech, K. J. 2001, BAAS, 33, no. 3, abstract no. 20.05

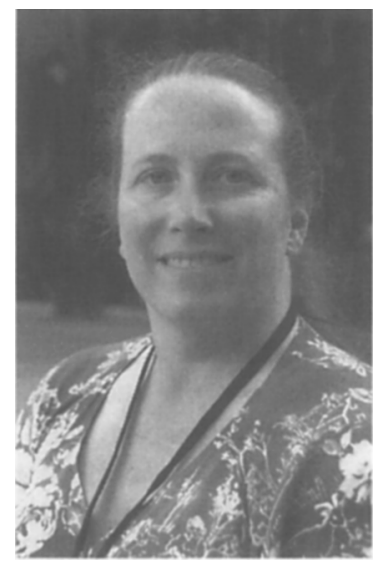

Karen Meech (photo: Seth Shostak) 